\title{
Modeling the indoor MIMO wireless channel
}

Michael A. Jensen

jensen@byu.edu

Jon W. Wallace

wall@ieee.org

Follow this and additional works at: https://scholarsarchive.byu.edu/facpub

Part of the Electrical and Computer Engineering Commons

\section{Original Publication Citation}

Wallace, J. W., and M. A. Jensen. "Modeling the Indoor MIMO Wireless Channel." Antennas and Propagation, IEEE Transactions on 5.5 (22): 591-9

\section{BYU ScholarsArchive Citation}

Jensen, Michael A. and Wallace, Jon W., "Modeling the indoor MIMO wireless channel" (2002). Faculty Publications. 542.

https://scholarsarchive.byu.edu/facpub/542

This Peer-Reviewed Article is brought to you for free and open access by BYU ScholarsArchive. It has been accepted for inclusion in Faculty Publications by an authorized administrator of BYU ScholarsArchive. For more information, please contact ellen_amatangelo@byu.edu. 


\title{
Modeling the Indoor MIMO Wireless Channel
}

\author{
Jon W. Wallace, Student Member, IEEE, and Michael A. Jensen, Senior Member, IEEE
}

\begin{abstract}
This paper demonstrates the ability of a physically based statistical multipath propagation model to match capacity statistics and pairwise magnitude and phase distributions of measured $4 \times 4$ and $10 \times 10$ narrow-band multiple-input multiple-output data (MIMO) at $2.4 \mathrm{GHz}$. The model is compared to simpler statistical models based on the multivariate complex normal distribution with either complex envelope or power correlation. The comparison is facilitated by computing channel element covariance matrices for fixed sets of multipath statistics. Multipolarization data is used to demonstrate a simple method for modeling dual-polarization arrays.
\end{abstract}

Index Terms-Channel models, indoor channels, measured channel data, multiple-input multiple-output (MIMO) channels, polarization.

\section{INTRODUCTION}

$\mathbf{R}$ ECENT STUDIES have demonstrated the impressive theoretical capacity of wireless systems operating in a multipath environment and employing multiple antennas on both transmit and receive [1]-[4]. These multiple-input multiple-output (MIMO) systems must cleverly exploit the structure of the channel transfer matrix (denoted as $\mathbf{H}$ ) to maximize data throughput. Accurate models that capture the complex spatial behavior of the propagation channel facilitate the development of these MIMO systems.

Many avenues exist for modeling the MIMO channel. For example, simple analytical models have initially been employed to understand possible gains from the MIMO channel [1]-[3]. Although advantageous for closed-form derivation of various channel parameters, these simple models often fail to capture the behavior of real channels. Alternately, direct measurement provides an exact characterization of $\mathbf{H}$ for the specific measurement scenario [5]-[9], and empirical statistical models may be developed based on an ensemble of measurements. However, applicability of such models may be limited to the specific array configuration or propagation environment under test. Deterministic physical models such as ray tracing [10], [11] simulate specific propagation scenarios and may be combined with Monte Carlo analysis to provide statistical channel information. Such methods promise an accurate characterization of the channel at the expense of computational resources. Finally, physically based statistical models [12]-[14] derive channel behavior from basic principles of radio propagation. The necessary channel parameters are then obtained by fitting the models to measured data. Such models are attractive since they are applicable to

Manuscript received June 7, 2001; revised December 21, 2001. This work was supported in part by the National Science Foundation under Wireless Initiative Grant CCR 99-79452 and in part by the Information Technology Research Grant CCR-0081476.

The authors are with the Wireless Research Group, Brigham Young University, Provo, UT 84602-4099 USA (e-mail: wall@ieee.org; jensen@ee.byu.edu).

Publisher Item Identifier S 0018-926X(02)05452-2.
TABLE I

Parameters Describing the MeAsured DATA SETS COLLECTED FOR THIS WORK

\begin{tabular}{llll}
\hline Parameter & Set 1 & Set 2 & Set 3 \\
\hline Antennas & $4 \times 4$ & $10 \times 10$ & $2 \times 2$ \\
Carrier Freq. & $2.45 \mathrm{GHz}$ & $2.42 \mathrm{GHz}$ & $2.45 \mathrm{GHz}$ \\
Modulation & BPSK & BPSK & BPSK \\
Symbol Rate & $12.5 \mathrm{~kb} / \mathrm{s}$ & $12.5 \mathrm{~kb} / \mathrm{s}$ & $12.5 \mathrm{~kb} / \mathrm{s}$ \\
Antenna Sep. & $\lambda / 2$ & $\lambda / 4$ & $\lambda / 2$ \\
Antenna Type & Patch & Monopole & Patch \\
Polarization & $\mathrm{V}$ & $\mathrm{V}$ & $\mathrm{V} / \mathrm{H}$ \\
\hline
\end{tabular}

many different array geometries and propagation environments and require modest computational resources.

In this paper, we employ a physical model that statistically describes the time of arrival (TOA), angle of arrival (AOA), and angle of departure (AOD) of each multipath component [12], [13]. We show that this model can match capacity, joint magnitude, and phase probability density functions (pdfs) of measured data for realistic model parameters. We also assess the applicability of simpler multivariate complex normal models based on power correlation and complex envelope correlation. Finally, we present a simple polarization model based on indoor dual-polarized measurements.

\section{Measured Channel Data}

For this study, MIMO channel data was collected on the fourth floor of the engineering building on the Brigham Young University campus [5], [6]. This measurement platform is able to measure the MIMO channel transfer matrix for up to 16 transmit and 16 receive antenna channels. The center frequency for measurements is tunable within the lower microwave bands, although all measurements presented here have been performed near $2.45 \mathrm{GHz}$. The system modulates (binary phase shift keyed or BPSK) the signals for each transmit antenna using a unique binary code sequence and the channel matrix is then estimated at the receiver using a maximum-likelihood algorithm. Table I lists the measurement parameters for the data sets under consideration.

Set 1 contains $4 \times 4$ data from five different scenarios. In each scenario, the transmitter was fixed in one room, while the receiver was moved to several different locations in another room. Since the rooms shared a wall only in one scenario, the data set exhibited fairly rich multipath interference.

In Set 2, the receive array assumed six possible positions in one room, and the transmit array assumed four possible positions in another nonadjacent room. Every combination of transmit and receive position was measured. Due to wide separation of transmit and receive, this set also exhibited rich multipath interference. 
In Set 3, the effect of polarization was explored using arrays with two dual-polarization patches separated by $\lambda / 2$. The transmitter was placed in a hallway at six different locations. The receiver was placed in a room off of this hallway in six other locations. Each possible combination of locations for transmit and receive was probed. The transmit and receive patch arrays faced each other in each case, and therefore a strong line-of-sight (LOS) path was present.

\section{Channel Model Preliminaries}

There are several important issues relevant to modeling the MIMO wireless channel. For this discussion, the $N_{R}$ receive by $N_{T}$ transmit narrowband-channel matrix $\mathbf{H}$ relates the transmit (x) and receive (y) complex baseband vectors as

$$
\mathbf{y}=\mathbf{H x}+\boldsymbol{\eta}
$$

where $\eta$ is the independent and identically distributed (i.i.d.) complex white Gaussian receiver noise vector.

\section{A. Channel Normalization}

Obtaining a good statistical sample of the indoor channel requires collecting data in a variety of scenarios. Large movement in transmit and receive location leads to substantial change in the bulk path loss of propagating signals. Effects of path loss can easily overshadow interesting channel behavior such as spatial correlation of transmit and receive signals. One way to remove this effect from collected data is to normalize the channel matrices.

Unless otherwise specified, channel matrices were normalized to force unit average single-input single-output (SISO) gain. The individual receiver noise is then given as $\sigma^{2}=P_{T} / \mathrm{SNR}$, where $P_{T}$ is the total transmit power and SNR represents the desired signal-to-noise ratio at the receiver. This normalization is equivalent to specifying the average receiver SNR when transmit streams are uncorrelated. The normalization constant may be computed for each individual $\mathbf{H}$ matrix or over all $\mathbf{H}$ matrices at a single location. In this paper, the normalization was computed on each $\mathbf{H}$ matrix for capacity and over all $\mathbf{H}$ matrices at a location for other quantities.

Removal of channel path loss is justified for modeling the subtle effects of multipath propagation. Realistic models should include path loss as a bulk signal attenuation which varies with separation of transmit and receive. When comparing various transmission schemes (e.g., dual polarization, directional antennas), care also must be taken that normalization does not force unwarranted conclusions.

\section{B. Capacity}

In this paper, capacity is computed by normalizing channel matrices to obtain an average SISO SNR of $20 \mathrm{~dB}$. Capacity is computed using the water-filling solution on the channel orthogonalized with the singular value decomposition (see [2], [15]).

\section{Joint pdfs}

The complete joint probability density function (pdf) for all elements of the $\mathbf{H}$ matrix provides a complete statistical description of the narrowband MIMO channel. If sufficient data were collected, one could compare measured and modeled channels by appropriately sampling this multidimensional pdf. However, as the number of antennas on transmit and receive increases, the dimensionality of the pdf becomes prohibitive and marginal pdfs or statistical moments must be used instead.

As a first step toward comparison of measured and modeled channels, we use pairwise joint pdfs on magnitude and phase. We concentrate specifically on the statistics of adjacent elements at transmit and receive, since these will be the most correlated. The measured bivariate pdf for adjacent transmit/receive element magnitude is

$$
f_{m P}\left(\left|x_{1}\right|,\left|x_{2}\right|\right)=\operatorname{HIST}_{\{i, j, k, \ell\} \in m_{P}}\left(\left|H_{i j}\right|,\left|H_{k \ell}\right|\right)
$$

where $P \in\{T, R\}$ for transmit or receive

$$
m_{T}=\{i, j, k, \ell: \ell=j+1, k=i\}, m_{R}=\{i, j, k, \ell: \ell=j, k=i+1\}
$$

and HIST2 is a two-dimensional (2-D) normalized histogram operation. The measured univariate pdf for adjacent transmit/receive element phase difference is given as

$$
f_{p P}(\Delta \phi)=\operatorname{HIST}_{\{i, j, k, \ell\} \in m_{P}}\left[\arg \left(H_{k \ell \ell} / H_{i j}\right)\right]
$$

where HIST is a one-dimensional (1-D) normalized histogram operation.

\section{Multivariate Complex Normal Distribution}

The multivariate complex normal distribution is fundamental to the study of the various models. Aspects relevant to this study are presented here for convenience.

1) Joint $p d f$ : The joint multivariate complex normal distribution [16] is given as

$$
f(\mathbf{x})=\frac{1}{\pi^{N}|\mathbf{R}|} \exp \left[-(\mathbf{x}-\boldsymbol{\mu})^{H} \mathbf{R}^{-1}(\mathbf{x}-\boldsymbol{\mu})\right]
$$

where $\mathbf{R}$ is the covariance matrix, $N$ is the dimensionality of $\mathbf{R}$, and $\boldsymbol{\mu}$ is the mean vector. The pairwise joint pdf $f\left(x_{m}, x_{n}\right)$ is given as (4) with $R$ replaced by the covariance submatrix $\mathcal{R}$, or

$$
\mathcal{R}=\left[\begin{array}{ll}
\mathcal{R}_{11} & \mathcal{R}_{12} \\
\mathcal{R}_{21} & \mathcal{R}_{22}
\end{array}\right]=\left[\begin{array}{ll}
R_{m m} & R_{m n} \\
R_{m n}^{*} & R_{m m}
\end{array}\right]
$$

where $R_{m m}=R_{n n}$ has been assumed.

2) Pairwise $p d f s$ : When $\boldsymbol{\mu}=\mathbf{0}$, the pairwise joint magnitude pdf is

$$
\begin{aligned}
f\left(r_{1}, r_{2}\right)= & \int_{2 \pi} \int_{2 \pi} f\left(x_{m}, x_{n}\right) d \phi_{1} d \phi_{2} \\
= & \frac{4 r_{1} r_{2}}{\Delta} \exp \left[\frac{-\mathcal{R}_{11}\left(r_{1}^{2}+r_{2}^{2}\right)}{\Delta}\right] \\
& \times J_{0}\left(-2 j \frac{\left|\mathcal{R}_{12}\right| r_{1} r_{2}}{\Delta}\right)
\end{aligned}
$$


where $x_{m}=r_{1} \exp \left(j \phi_{1}\right), x_{n}=r_{2} \exp \left(j \phi_{2}\right)$, and $\Delta=\mathcal{R}_{11}^{2}-$ $\left|\mathcal{R}_{12}\right|^{2}$. The pdf for pairwise phase difference is

$$
\begin{aligned}
f(\Delta \phi) & =\int_{0}^{\infty} \int_{0}^{\infty} \int_{2 \pi} f\left(x_{m}, x_{n}\right) d \phi_{1} d r_{1} d r_{2} \\
& =\frac{\chi^{1 / 2}+2 b\left[\tan ^{-1}\left(\frac{b}{\chi^{1 / 2}}\right)-\tan ^{-1}\left(\frac{2 a+b}{\chi^{1 / 2}}\right)\right]}{(\pi \Delta / 2) \chi^{3 / 2}}
\end{aligned}
$$

where

$\chi=4 a^{2}-b^{2}, a=-\mathcal{R}_{11} / \Delta, b=\left(2\left|\mathcal{R}_{12}\right| / \Delta\right) \cos \left(\Delta \phi \operatorname{targ}\left\{\mathcal{R}_{12}\right\}\right)$

and in this case we express $x_{n}$ as $x_{n}=r_{2} \exp \left[j\left(\phi_{1}+\Delta \phi\right)\right]$. Averaging the pdfs associated with all element pairs for a given transmit and receive spacing results in an average pairwise pdf, which is analogous to those given in Section III-C.

3) Covariance Matrices and Simplifying Assumptions: The zero mean multivariate complex normal distribution is completely characterized by the covariance matrix $\mathbf{R}$. For the purpose of modeling $\mathbf{H}$, the $\left(N_{T} N_{R}\right) \times\left(N_{T} N_{R}\right)$ covariance matrix is defined as

$$
R_{i j, k \ell}=E\left\{H_{i j} H_{k \ell \ell}^{*}\right\}
$$

where $i$ and $j$ combine to form a row index of $\mathbf{R}$ and $k$ and $\ell$ combine to form a column index of $\mathbf{R}$. A number of assumptions are convenient when working with the covariance matrix. Separability assumes that the full covariance matrix may be written as a product of transmit covariance $\left(\mathbf{R}_{\mathbf{T}}\right)$ and receive covariance $\left(\mathbf{R}_{\mathbf{R}}\right)$ or

$$
R_{i j, k \ell}=R_{R, i k} R_{T, j \ell} .
$$

For such channels, the transmit and receive covariance matrices can be computed from the full covariance matrix as

$$
\begin{aligned}
& R_{T, i j}=\frac{1}{\alpha} \sum_{k=1}^{N_{R}} R_{k i, k j} \\
& R_{R, i j}=\frac{1}{\beta} \sum_{k=1}^{N_{T}} R_{i k, j k}
\end{aligned}
$$

where $\alpha$ and $\beta$ are chosen such that

$$
\alpha \beta=\sum_{k: 1=1}^{N_{R}} \sum_{k: 2=1}^{N_{T}} R_{k_{1} k_{2}, k_{1} k_{2}} .
$$

In the case where $\mathbf{R}$ is a correlation coefficient matrix, we may choose $\alpha=N_{R}$ and $\beta=N_{T}$. Separability makes implications about the statistical independence of multipath fading due to transmit location and receive location.

Shift-invariance assumes that the covariance matrix is only a function of antenna spacing and not absolute antenna location. The relationship between the full covariance and shift-invariant covariance $\left(\mathbf{R}^{\mathbf{S}}\right)$ is

$$
R_{i j, k \ell}=R_{i-k, j-\ell}^{S}
$$
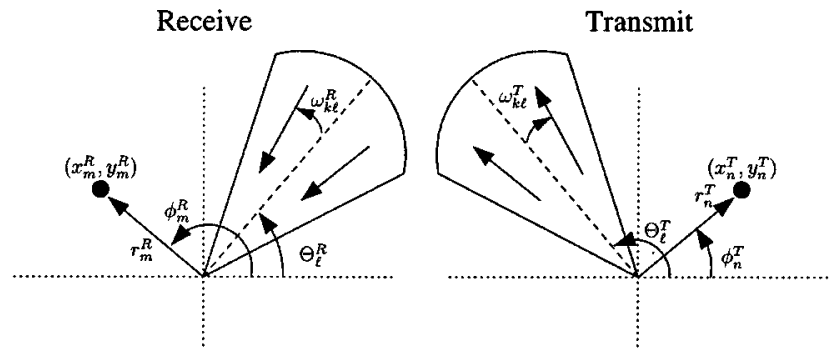

Fig. 1. Transmit and receive parameters for a single cluster in the SVA model.

The combination of separability and shift-invariance allows full covariance matrices to be generated from existing correlation functions, which relate correlation to receive element displacement. For example, we may use Jakes' model to obtain

$$
R_{i j, k \ell}=J_{0}\left[2 \pi\left|\mathbf{x}_{i}^{R}-\mathbf{x}_{k}^{R}\right|\right] J_{0}\left[2 \pi\left|\mathbf{x}_{j}^{T}-\mathbf{x}_{\ell}^{T}\right|\right]
$$

where $\mathrm{x}_{i}^{\{T, R\}}$ is the vectorial location of the $i$ th transmit or receive antenna in wavelengths, and $|\cdot|$ is the vector norm.

4) Computer Generation: Computer generation of zero mean complex normal vectors for a specified covariance matrix $\mathbf{R}$ is performed by generating vectors of i.i.d. complex normal elements with unit variance (a). The transformation $\mathbf{y}=\boldsymbol{\Sigma} \boldsymbol{\Lambda}^{1 / 2} \mathbf{a}$ (where $\boldsymbol{\Sigma}$ and $\boldsymbol{\Lambda}$ are the matrix of eigenvectors and the diagonal matrix of eigenvalues of $\mathbf{R}$, respectively) yields a complex normal vector $(\mathbf{y})$ with the proper correlation structure.

\section{Saleh-Valenzuela Model With AOA/AOD}

This section demonstrates that an extension of the Saleh-Valenzuela model [12] that includes AOA statistics [13] is able to match capacity pdfs and pairwise element pdfs of the measured channel. Here, AOD statistics are assumed to follow the same distribution as AOA, which is reasonable for the indoor channel with the same basic configuration on transmit and receive. We refer to the Saleh-Valenzuela model with AOA/AOD as the SVA model for brevity.

The SVA model characterizes the channel by representing each multipath component in terms of its amplitude, arrival time, and AOA/AOD. Based upon experimental observations, these arrivals or rays arrive in clusters in both space and time. Fig. 1 shows the model parameters for a single cluster in the SVA model. The directional channel impulse response arising from $L$ clusters and $K$ rays per cluster is

$$
\begin{array}{r}
h\left(\theta^{R}, \theta^{T}\right)=\frac{1}{\sqrt{L K}} \sum_{\ell=0}^{L-1} \sum_{k=0}^{K-1} \beta_{k \ell} \delta\left(\theta^{T}-\Theta_{\ell}^{T}-\omega_{k \ell}^{T}\right) \\
\times \delta\left(\theta^{R}-\Theta_{\ell}^{R}-\omega_{k \ell}^{R}\right)
\end{array}
$$

where $\theta^{T}$ and $\theta^{R}$ are transmit and receive angles, $\beta_{k \ell}$ is the complex ray gain, $\Theta_{\ell}^{T}$ and $\Theta_{\ell}^{R}$ are the mean transmit and receive angles within the $\ell$ th cluster, and $\omega_{k \ell}^{T}$ and $\omega_{k \ell}^{R}$ are the transmit and receive angles of the $k$ th ray in the $\ell$ th cluster, relative to the respective mean angles in each cluster. 
To simplify the model, average-ray power in each cluster is constant so that $\beta_{k \ell} \sim \mathcal{C N}\left(0,\left|\beta_{\ell}\right|^{2}\right)$, where $\mathcal{C N}\left(\mu, \sigma^{2}\right)$ denotes the complex normal distribution with mean $\mu$ and variance $\sigma^{2}$. The cluster amplitude is Rayleigh distributed with the expected cluster power (or variance) satisfying $E\left\{\left|\beta_{\ell}\right|^{2}\right\}=$ $\exp \left(-T_{\ell} / \Gamma\right)$, where $T_{\ell}$ is the arrival time of the $\ell$ th cluster, and $\Gamma$ is the cluster decay time constant. The arrival time distribution is a conditional exponential with a normalized unit arrival rate. Details concerning the model implementation can be found in [12], [13], [17]. The notation $\operatorname{SVA}(\Gamma, \sigma)$ is used in this paper to denote the SVA model with constant average ray power and unit cluster arrival rate, where $\Gamma$ is the cluster decay constant and $\sigma$ is the standard deviation of ray AOA/AOD.

The narrow-band channel matrix is computed from the directional impulse response as

$$
h_{m n}=\int_{2 \pi} \int_{2 \pi} W_{m}^{R}\left(\theta^{R}\right) h\left(\theta^{R}, \theta^{T}\right) W_{n}^{T}\left(\theta^{T}\right) d \theta^{T} d \theta^{R}
$$

where $W_{q}^{P}(\theta)=g_{q}^{P}(\theta) \exp \left[j \psi_{q}^{P}(\theta)\right], g_{q}^{P}(\theta)$ is the antenna gain pattern, $\psi_{q}^{P}(\theta)=2 \pi\left[x_{q}^{P} \cos (\theta)+y_{q}^{P} \sin (\theta)\right], P \in\{T, R\}$, and $q \in\{m, n\}$. Based upon measured data taken in [13], a twosided Laplacian distribution is assumed for the ray AOA/AOD distribution whose pdf is

$$
f^{P}(\omega)=\frac{1}{\sqrt{2} \sigma_{P}} \exp \left(-\left|\sqrt{2} \omega / \sigma_{P}\right|\right)
$$

where $\sigma_{P}$ is the standard deviation of angle in radians.

\section{A. Complex Normal Approximation}

H matrices may be generated directly by computing (16) for each realization of the SVA model. An alternate method computes channel matrices according to a complex normal distribution for each fixed set of cluster statistics. This method reduces computational time and links the model to simpler complex normal models.

For a fixed set of cluster statistics $\left(\Theta_{\ell}^{T}, \Theta_{\ell}^{R},\left|\beta_{\ell}\right|^{2}\right)$ and ray arrival angles $\left(\omega_{k \ell \ell}^{T}, \omega_{k \ell}^{R}\right), h_{m n}$ is a weighted sum of zero mean complex normal random variables, resulting in a correlated complex normal distribution. If the angular spread on $\omega$ is small, the $h_{m n}$ will look closely complex normal even if the $\omega_{k \ell}^{P}$ are allowed to vary. In this case, we find the average covariance matrix $\overline{\mathbf{R}}$ as

$$
\begin{aligned}
& \bar{R}_{m_{1} n_{1}, m_{2} n_{2}} \\
& =E\left\{h_{m_{1}, n_{1}} h_{m_{2}, n_{2}}^{*}\right\} \\
& =\frac{1}{K L} \sum_{\ell_{1}=0}^{L-1} \sum_{\ell_{2}=0}^{L-1} \sum_{k_{1}=0}^{K-1} \sum_{k_{2}=0}^{K-1} E\left\{\beta_{k_{1} \ell_{1}} \beta_{k_{2} \ell_{2}}^{*}\right\} \\
& \quad \times E\left\{W_{m_{1}}^{R}\left(\Theta_{\ell_{1}}^{R}+\omega_{k_{1} \ell_{1}}^{R}\right) W_{m_{2}}^{R *}\left(\Theta_{\ell_{2}}^{R}+\omega_{k_{2} \ell_{2}}^{R}\right)\right\} \\
& \quad \times E\left\{W_{n_{1}}^{T}\left(\Theta_{\ell_{1}}^{T}+\omega_{k_{1} \ell_{1}}^{T}\right) W_{n_{2}}^{T *}\left(\Theta_{\ell_{2}}^{T}+\omega_{k_{2} \ell_{2}}^{T}\right)\right\}
\end{aligned}
$$

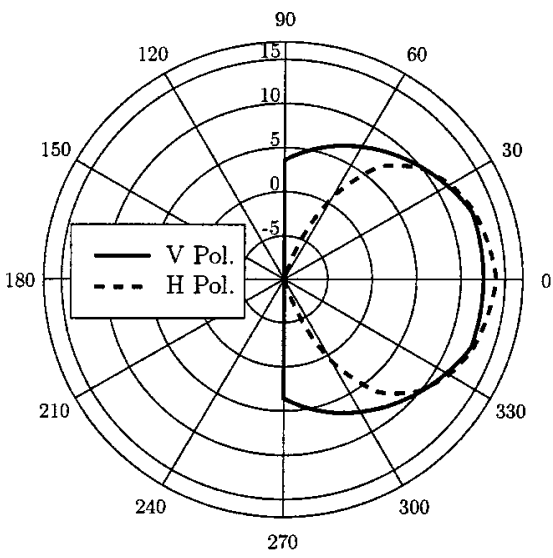

Fig. 2. Radiated power $(\mathrm{dB})$ for vertical/horizontal polarized patch antenna relative to a uniform radiator, as a function of azimuth angle.

where statistical independence of complex ray gain, AOA, and AOD has been assumed. If the gains of distinct rays are independent and ray $\mathrm{AOA} / \mathrm{AOD}$ are i.i.d., the expression simplifies to

$$
\bar{R}_{m_{1} n_{1}, m_{2} n_{2}}=\frac{1}{L} \sum_{\ell}\left|\beta_{\ell}\right|^{2} L_{m_{1} m_{2}}^{R}\left(\Theta_{\ell}^{R}\right) L_{n_{1} n_{2}}^{T}\left(\Theta_{\ell}^{T}\right)
$$

where

$$
\begin{aligned}
L_{q_{1} q_{2}}^{P}\left(\Theta_{\ell}^{P}\right)=\int f^{P}(\omega) g_{q_{1}}^{P}\left(\Theta_{\ell}^{P}+\omega\right) g_{q_{2}}^{P *}\left(\Theta_{\ell}^{P}+\omega\right) \\
\times \exp \left[j \psi_{q_{1} q_{2}}^{P}\left(\Theta_{\ell}^{P}+\omega\right)\right] d \omega
\end{aligned}
$$

$f^{P}(\omega)$ is the ray angle of arrival/departure pdf

$\psi_{q_{1} q_{2}}^{P}(\theta)=2 \pi r_{q_{1} q_{2}}^{P} \cos \left(\theta-\phi_{q_{1} q_{2}}^{P}\right), r_{q_{1} q_{2}}^{P}=\left[\left(x_{q_{1}}^{P}-x_{q_{2}}^{P}\right)^{2}+\left(y_{q_{1}}^{P}-y_{q_{2}}^{P}\right)^{2}\right]^{1 / 2}$

and

$$
\phi_{q_{1} q_{2}}^{P}=\tan ^{-1}\left[\left(y_{q_{1}}^{P}-y_{q_{2}}^{P}\right) /\left(x_{q_{1}}^{P}-x_{q_{2}}^{P}\right)\right] .
$$

For certain special cases, closed-form expressions for (20) exist. For arbitrary antenna gain and angular ray distributions, however, (20) is computed numerically. The result is a relatively simple expression for the mean channel covariance matrix for a fixed set of cluster statistics. We note that although the covariance matrix given by (19) is not strictly separable (Section III-D3) for a single cluster realization, it approaches separability when averaged over many random cluster realizations where transmit and receive statistics are independent. Also, assuming a uniform linear array with one gain pattern for all transmit elements and another for all receive elements results in a shift-invariant covariance matrix.

\section{B. Comparison of Model and Data}

In [13], high-resolution AOA measurements were performed on the same floor of the BYU engineering building as in this study. Although the measurements were at a much higher frequency $(\approx 7 \mathrm{GHz})$, the extracted parameters serve as a logical starting point. The key parameters are (see [13]) $\sigma_{\{T, R\}}=$ $26^{\circ}, \Gamma=2, \Lambda=1$. For simulation, transmit and receive cluster arrival angles are assumed to be uniform on $[0,2 \pi]$. 

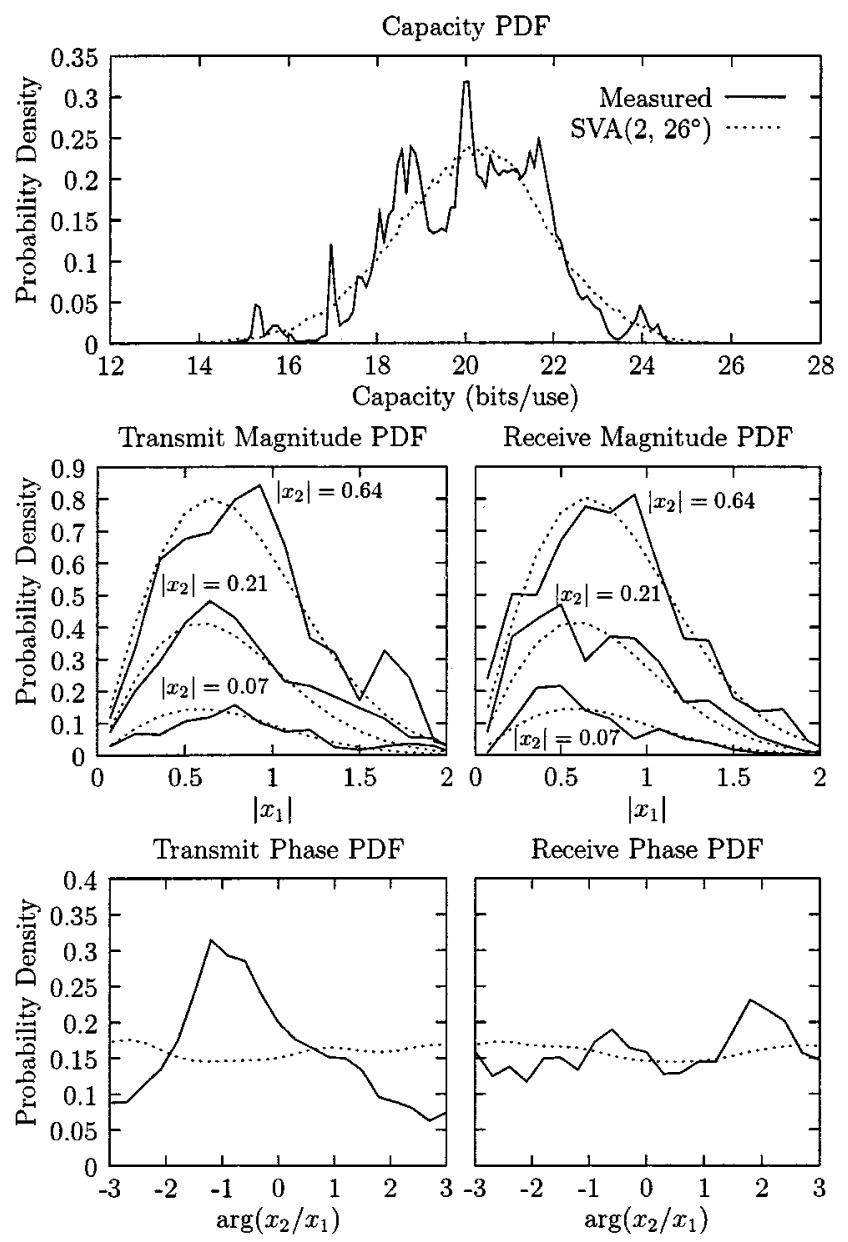

Fig. 3. Comparison of capacity pdfs and joint magnitude and phase pdfs for $4 \times 4$ measured data and SVA model simulations.

1) $4 \times 4$ Data: First, capacity pdfs and pairwise pdfs from the model are compared with measured $4 \times 4$ data from Set 1 . Fig. 2 shows the approximate gain pattern for the vertically polarized patch antenna obtained using a piecewise linear fit to the output of moment method simulations. This gain pattern is required to compute (20).

Fig. 3 compares pdfs of measured data and Monte Carlo simulations of the SVA model. In these and later simulations, $10^{\tilde{z}}$ channels were realized (100 cluster configurations with 1000 channels each). PDFs were computed by averaging (6) and (7) for magnitude and phase over the 100 cluster configurations.

Apparent in the figure is the good fit of both the capacity pdfs and pairwise amplitude pdfs. The discrepancy in phase is due to two basic factors: 1) imperfect phase response of hardware for the transmit and receive channels and 2) the uniform cluster $\mathrm{AOA} / \mathrm{AOD}$ assumption is not strictly valid over the limited data set.

2) $10 \times 10$ Data: Next, capacity pdfs and pairwise pdfs from the model are compared with measured $10 \times 10$ data from Set 2. This data set employed quarter-wave monopole antennas, and an ideal uniform radiation pattern in azimuth was assumed. Fig. 4 compares the pdfs for the measured and simulated $10 \times 10$ channel. Since the parameters from [13] were taken at a higher frequency and represent an average over many scenarios, the slight disagreement in the capacity curves is not surprising. The discrepancy suggests that the multipath in the measured environment is less than that specified in the simulation.

The amount of multipath in the simplified SVA model is controlled by the parameters $\Gamma$ (the cluster decay time constant) and $\sigma$ (the mean angular deviation of the rays in the clusters). Decreasing $\Gamma$ leads to fewer clusters and, therefore, less multipath. Similarly, decreasing $\sigma$ generates less isotropic multipath, limiting the ability of the arrays to exploit multiple rays within a cluster. As shown in the figure, lowering either of these parameters improves the agreement. However, ultimate validation of the model requires detailed AOA/AOD measurements at the 2.4-GHz carrier.

Both amplitude and phase pdfs are fairly insensitive to the parameter adjustments, suggesting that the multipath is at a level of saturation when considering just two closely spaced elements. The agreement of measured and simulated amplitude pdfs is fairly good. The disagreement in the phase pdfs, however, is likely due to the same problems mentioned in the $4 \times 4$ case.

\section{JoINT COMPLEX NoRMAL MOdels}

The multivariate complex normal distribution can be used to model the channel matrix directly by simply specifying the channel element covariance matrix. The wealth of correlation information provided by antenna diversity studies makes this approach particularly attractive. This section assesses the ability of complex envelope and power correlation models to match the capacity pdfs and pairwise magnitude and phase pdfs of the SVA model. The reason for using the SVA model as opposed to measured data is that the underlying covariance behavior is known and that unlimited channels may be generated.

\section{A. Complex-Envelope Method}

This method assumes that the underlying distribution on $\mathbf{H}$ is multivariate complex normal and specifies a covariance matrix (R) which is the average covariance of the true distribution or $\mathbf{R}=E\left\{\mathbf{h h}^{H}\right\}$, where $\mathbf{h}$ is a stacked channel matrix. Once the channel covariance matrix is known, the method in Section III-D4 is used to generate $\mathbf{H}$ matrices.

\section{B. Power-Covariance Method}

In this method, the channel matrices are computed as in Section V-A except that the covariance matrix is derived from the power-covariance matrix given as

$$
R_{i j, k \ell}^{P}=E\left\{\left|H_{i j}\right|^{2}\left|H_{k \ell}\right|^{2}\right\}-E\left\{\left|H_{i j}\right|^{2}\right\} E\left\{\left|H_{k \ell}\right|^{2}\right\} .
$$

as suggested in [18]. The same power covariance behavior can be generated using a zero mean multivariate complex normal distribution with covariance matrix $R=\sqrt{R^{P}}$, where $\sqrt{\cdot}$ is elementwise square root. However, care is required since the root of the power covariance matrix is not necessarily positive definite. Under such circumstances, the method outlined in Section III-D4 cannot be used directly. In this study, however, root power covariance matrices generated by the SVA model were always positive definite. 


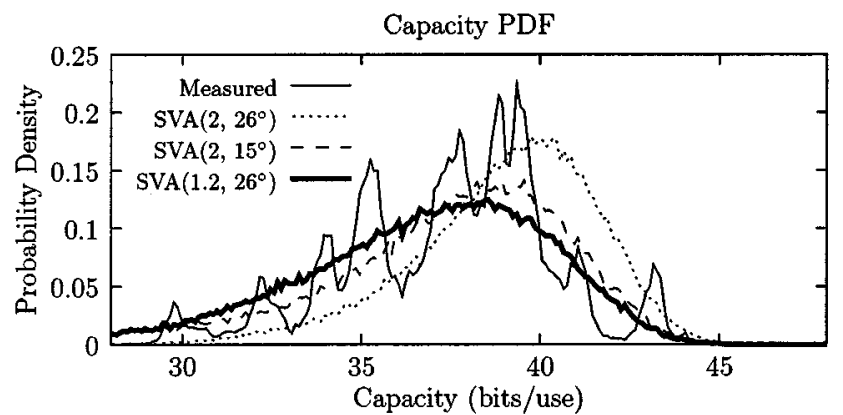

Transmit Magnitude PDF

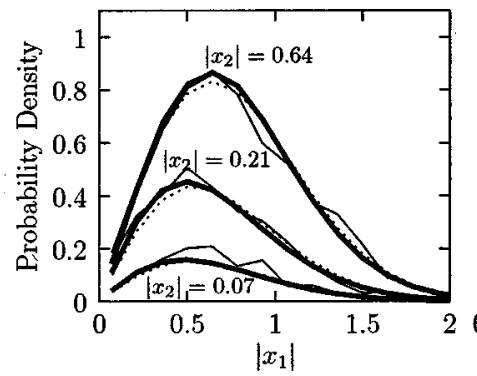

Receive Magnitude PDF
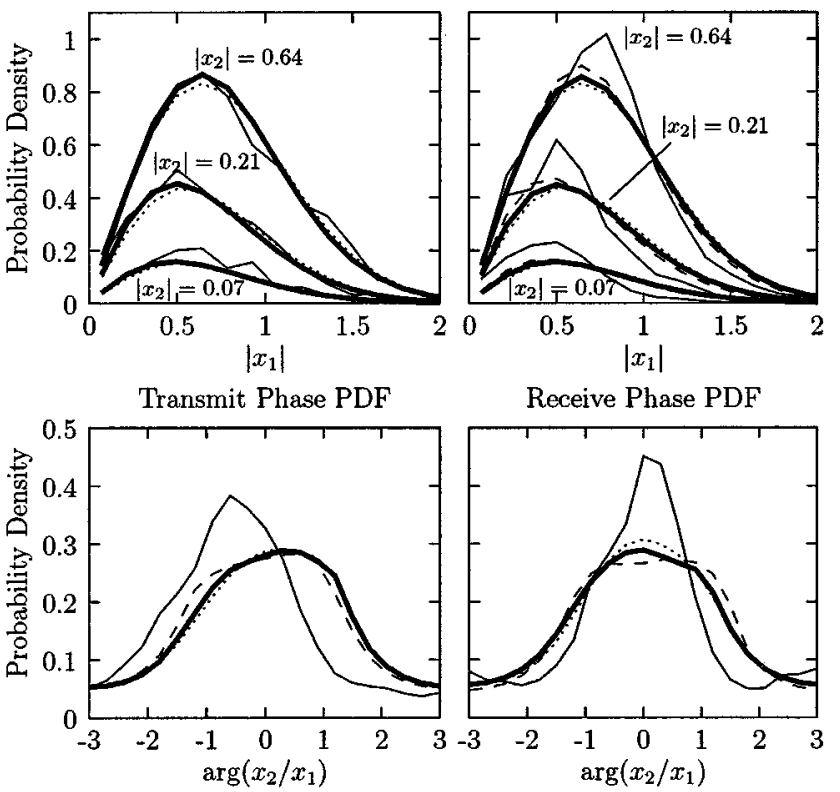

Receive Phase PDF

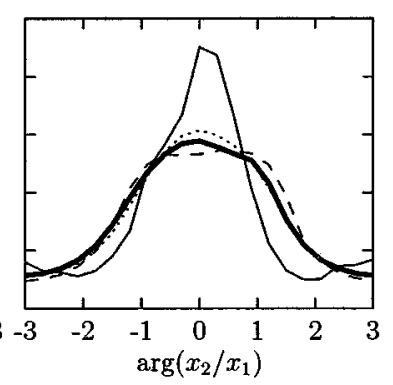

Fig. 4. Comparison of capacity pdfs and joint magnitude and phase pdfs for $10 \times 10$ measured data and SVA model simulations.

\section{Simulation Results}

Fig. 5 plots capacity pdfs and the average pairwise magnitude and phase pdfs for simulated $4 \times 4$ channel matrices. Since the pairwise pdfs for transmit and receive look nearly identical, they have been averaged to obtain one plot for magnitude and another for phase. Linear arrays were assumed with $\lambda / 2$ interelement spacing. Parameters for the SVA model were $\Gamma=2, \sigma=26^{\circ}$, and uniform cluster AOA/AOD. The complex envelope method exhibits a good match for the pairwise pdfs but overestimates capacity. The power correlation model matches capacity pdfs and magnitude pdfs better at the cost of ignoring phase.

Fig. 6 plots capacity pdfs and the average pairwise magnitude and phase pdfs for simulated $8 \times 8$ channel matrices with $\lambda / 2$ interelement spacing. The addition of antennas has apparently amplified the deficiencies present in the $4 \times 4$ case. Fig. 7 shows the performance of the two methods for $8 \times 8$ arrays with an interelement spacing of $\lambda / 4$. The complex envelope method performs about as well as the $\lambda / 2$ case. The power correlation method has great difficulty matching capacity, probably due to the significant correlation in phase, which is ignored.

The simple models fail to match the SVA model because the covariance matrix is constant only for a fixed set of cluster statistics. Fig. 8 demonstrates the random behavior of the covariance matrix by plotting the variance of the amplitude and phase of
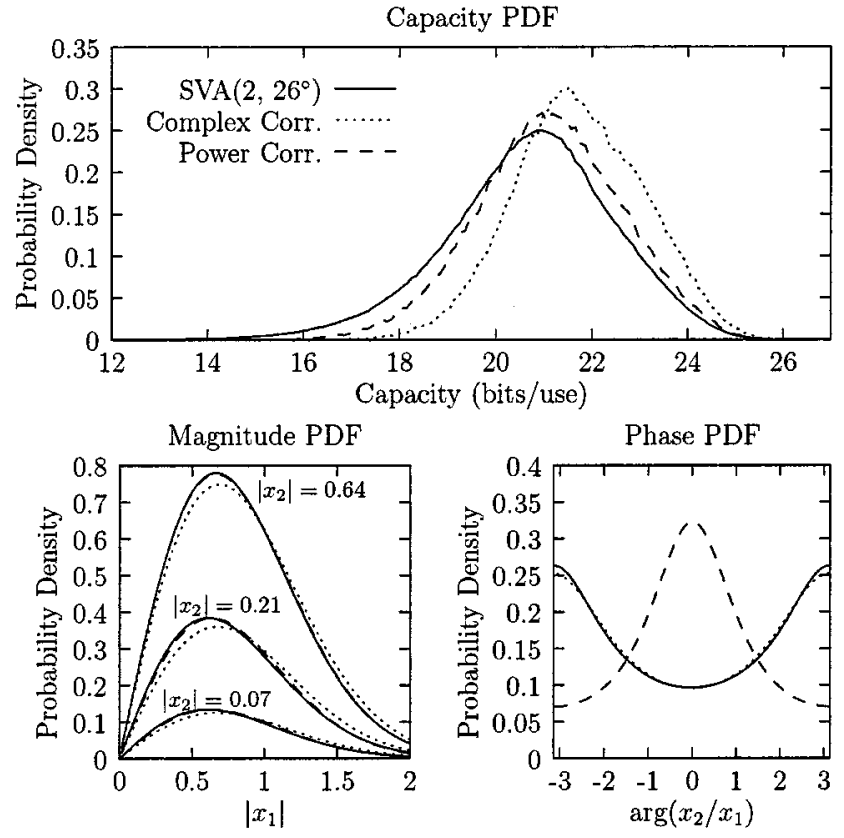

Fig. 5. Capacity pdfs and pairwise magnitude and phase pdfs for the $4 \times 4$ channel with $\lambda / 2$ interelement spacing.
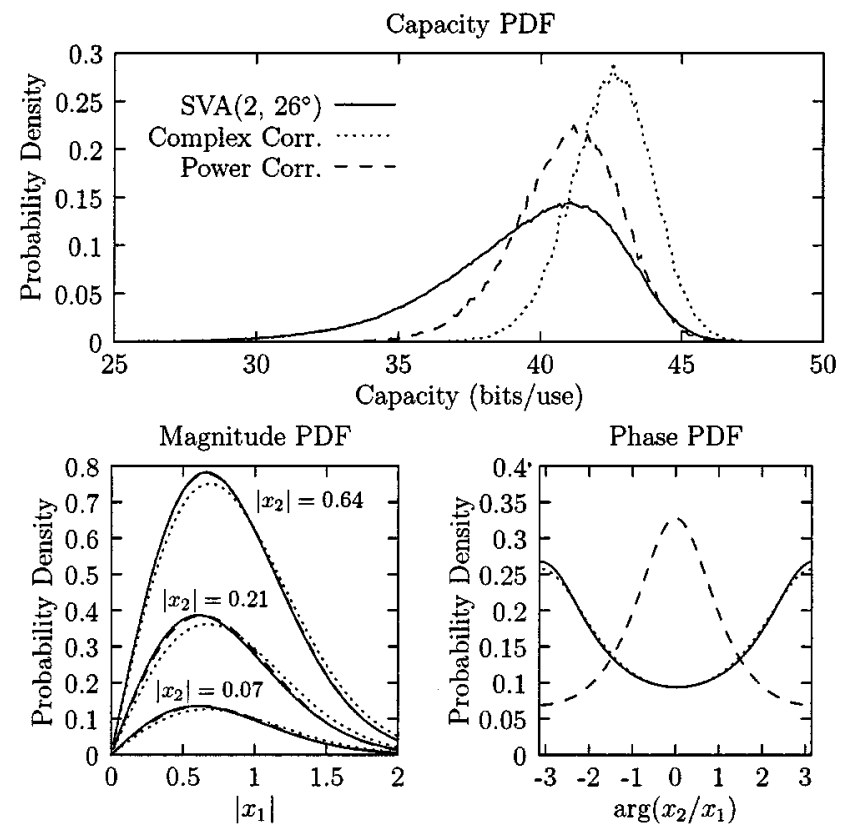

Fig. 6. Capacity pdf and pairwise magnitude and phase pdfs for $8 \times 8$ channel with $\lambda / 2$ interelement spacing.

the elements of the correlation coefficient matrix generated with SVA model for the two $8 \times 8$ cases. Shift invariance of the model has been assumed so that the correlation coefficients are only a function of antenna separation at transmit and receive. For $\lambda / 2$ separation, the element magnitudes (powers) and phases exhibit small and large variations, respectively. Low power variance and highly random phase seem to be a good candidate for a power correlation model. For the $\lambda / 4$ case, the power variation is more pronounced and the phases exhibit less variation. The poorer fit in capacity suggests that power models have difficulty in this case. 

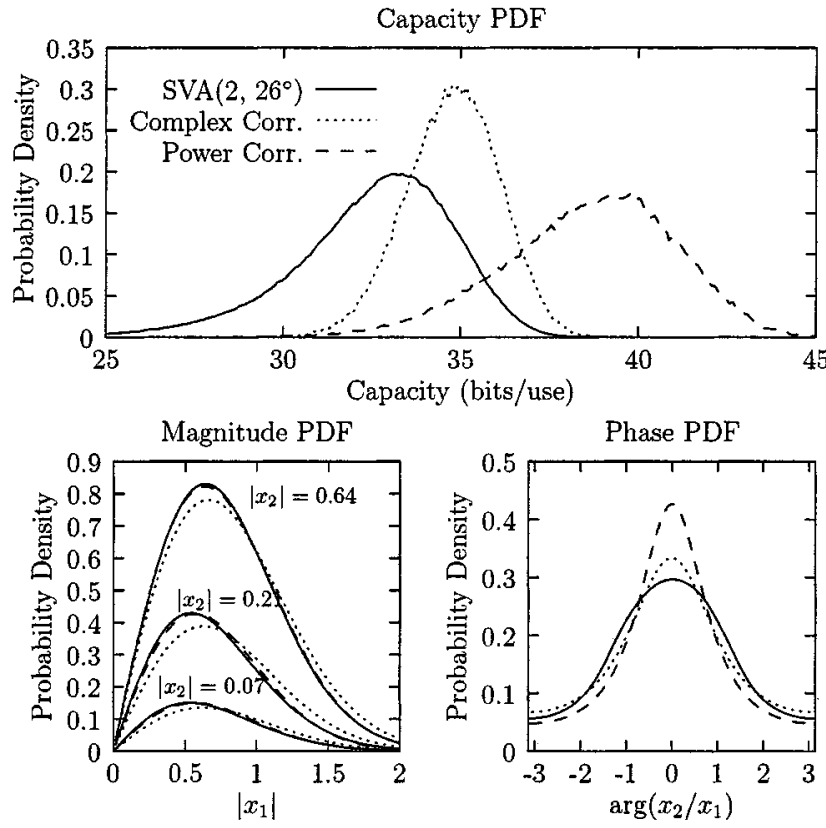

Fig. 7. Capacity pdf and pairwise magnitude and phase pdfs for the $8 \times 8$ channel with $\lambda / 4$ interelement spacing.
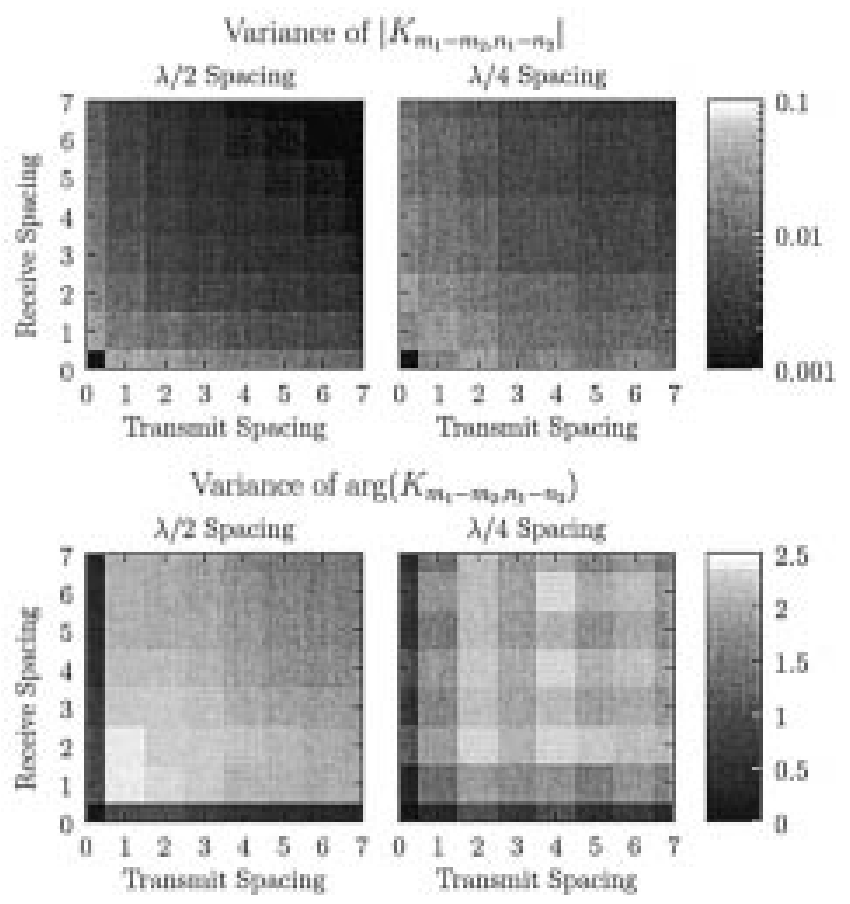

Fig. 8. Variance of the elements of the correlation coefficient matrix for the $8 \times 8$ channel data generated with the SVA model.

\section{Modeling of Multiple Polarizations}

Antenna elements employing multiple polarizations can increase capacity [19] and often require less space per transmit/receive channel than spatially separated single-polarization elements. The capacity performance of multipolarization elements is a function of the average depolarization ratio due to scattering in the transmission environment. Environments with low depolarization lead to nearly orthogonal channels at the expense of reduced average receiver SNR, whereas environments with high
TABLE II

Average Power Correlation of Subchannels Taken From NORMALIZED H MATRICES FROM DATA SET 3

\begin{tabular}{|c|c|c|c|c|}
\hline & $\overline{\mathrm{VV}}$ & $\overline{\mathrm{VH}}$ & $\overline{\mathrm{HV}}$ & $\overline{\mathrm{HH}}$ \\
\hline VV & 1.0000 & 0.0053 & -0.0563 & -0.2153 \\
\hline VH & 0.0053 & 1.0000 & 0.1164 & -0.0004 \\
\hline HV & -0.0563 & 0.1164 & 1.0000 & -0.0662 \\
\hline $\mathrm{HH}$ & -0.2153 & -0.0004 & -0.0662 & 1.0000 \\
\hline
\end{tabular}

depolarization behave similarly to spatially separated elements [20]. An analysis of the capacity of dual-polarization elements versus single-polarization elements is provided in [17]. In this paper, we outline a simple method for including polarization into existing single polarization models. The capacity statistics for measured $4 \times 4$ channels is matched using this method and the SVA model.

\section{A. Independent-Subchannel Method}

Pairwise magnitude and phase pdfs generated from data Set 3 show little dependence of both magnitude and phase for orthogonally polarized elements [17]. Table II lists the average power correlation coefficients for the various subchannels of the channel matrix. Negative correlations arise due to the channel normalization which is required due to the large variation in average receive power with large movement. Due to the small level of correlation for opposite polarizations, the various subchannels may initially be treated as statistically independent. Thus, we characterize the $\mathrm{VV}, \mathrm{HH}, \mathrm{VH}$, and $\mathrm{HV}$ channels in isolation and generate corresponding synthetic $\mathbf{H}$ matrices: $\mathbf{H}_{\mathbf{V V}}, \mathbf{H}_{\mathbf{H H}}, \mathbf{H}_{\mathbf{V H}}$, and $\mathbf{H}_{\text {IIV }}$. The complete channel is then formed as $\mathbf{H}=\left[\mathbf{H}_{\mathbf{V V}}, \alpha \mathbf{H}_{\mathbf{V H}} ; \alpha \mathbf{H}_{\mathbf{H V}}, \mathbf{H}_{\mathbf{H H}}\right]$. The constant $\alpha$ is chosen to ensure that the average depolarization ratio of the synthetic channel matrices matches that of the measured data.

\section{B. SVA-Model Parameters}

Due to the strong LOS nature of the scenario for Set 3, a reduction in the angular spread of arrivals within a cluster is expected, especially for the cluster corresponding to LOS. Also, transmit and receive patch antenna arrays were always facing each other, leading to a fixed mean cluster arrival angle for the LOS cluster. $\Gamma=2.0, \sigma=10^{\circ}$ were used for the copolarized subchannels $(\mathrm{VV} / \mathrm{HH})$ and $\Gamma=2.0, \sigma=20^{\circ}$ were used for the cross-polarized subchannels $(\mathrm{VH} / \mathrm{HV})$. The required increase in angular spread of the cross-polarized subchannels is reasonable due to stronger multiple reflections. The depolarization parameter $\alpha$ was chosen to match the measured average depolarization of $-6.8 \mathrm{~dB}$. Fig. 9 plots the capacity for the different simulated subchannels in isolation compared with the corresponding subchannels extracted from measured data. The sharp peak at the left of each capacity plot occurs where the water-filling solution uses only a single orthogonal subchannel, which happens frequently for these $2 \times 2$ channels exhibiting strong LOS. The sharpness of the peak results from the narrow bin size and the nearly constant gain of the strongest orthogonal subchannel taken from normalized $\mathbf{H}$. These plots reveal the 

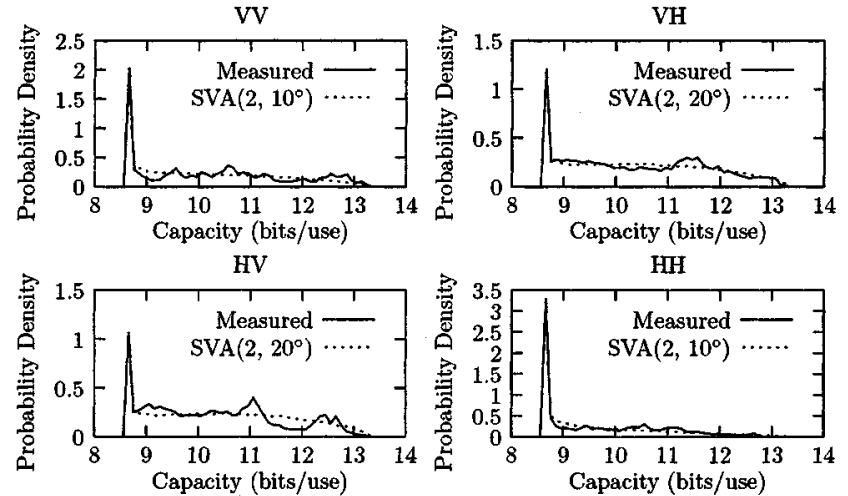

Fig. 9. Match of capacity pdfs for subchannels generated with the SVA model for various polarizations.
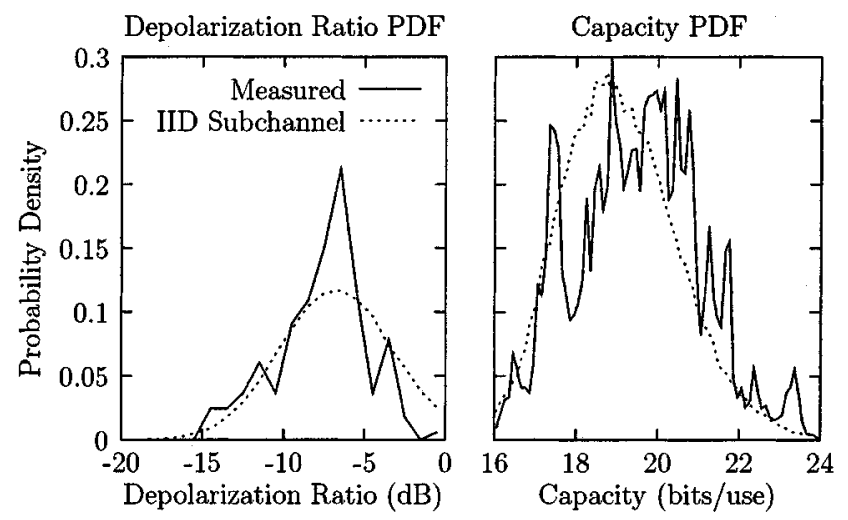

Fig. 10. Average depolarization ratio pdfs and capacity pdfs for measured and simulated channel matrices.

good statistical agreement between measured and modeled channels for the selected model parameters. This agreement suggests that the proposed mechanism for including polarization within the SVA model captures the channel behavior important for determining channel capacity.

\section{Simulation Results}

The SVA model was used to generate 100 cluster configurations with 1000 sets of $2 \times 2$ subchannel matrices each. The subchannel matrices were then formed into complete $4 \times 4$ channel matrices. Fig. 10 shows the depolarization pdfs and capacity pdfs for measured and simulated channel matrices. The fit in depolarization and capacity is good considering the simplicity of the model.

\section{CONCLUSION}

This paper has explored the ability of simple statistical models to capture key features of the narrow-band indoor MIMO wireless channel. Ultimately, a tradeoff exists between model complexity and accuracy. However, we have shown that even simple models (like the SVA model), which are based partially on channel physics, match capacity, and pairwise pdfs of measured data quite well. Models that ignore channel physics and attempt to force channel statistics to fit convenient distributions seem to have difficulty for increasingly complex channels. Thus, previous work in antenna diversity which focuses on bulk parameters like envelope and power correlation may have trouble finding direct application to MIMO channels. Also, we have provided a simple method for including polarization into existing models based on observations from measured dual-polarized data. Evaluation of space time coding algorithms and capacity studies should benefit from the simple modeling approaches presented in this work.

\section{REFERENCES}

[1] G. J. Foschini and M. J. Gans, "On limits of wireless communications in a fading environment when using multiple antennas," Wireless Personal Commun., vol. 6, no. 3, pp. 311-335, Mar. 1998.

[2] G. G. Rayleigh and J. M. Cioffi, "Spatio-temporal coding for wireless communication," IEEE Trans. Commun., vol. 46, pp. 357-366, Mar. 1998

[3] T. L. Marzetta and B. M. Hochwald, "Capacity of a mobile multiple-antenna communication link in Rayleigh flat fading," IEEE Trans. Inform. Theory, vol. 45, pp. 139-157, May 1999.

[4] G. Golden, C. Foschini, R. Valenzuela, and P. Wolniansky, "Detection algorithm and initial laboratory results using V -BLAST space-time communication architecture," Electron. Lett., vol. 35, no. 1, pp. 14-15, Jan. 1999.

[5] J. W. Wallace and M. A. Jensen, "Spatial characteristics of the MIMO wireless channel: Experimental data acquisition and analysis," in Proc. IEEE Intl. Conf. Acoustics, Speech, Signal Processing (ICASSP), vol. 4, Salt Lake City, UT, May 2001, pp. 774-779.

[6] - "Experimental characterization of the MIMO wireless channel," in Proc. IEEE Antennas Propagat. Intl. Symp. Dig. , Boston, MA, July 2001

[7] B. Jeffs, E. Pyper, and B. Hunter, "A wireless MIMO channel probing approach for arbitrary antenna arrays," IEEE Intl. Conf. Acoustics, Speech, Signal Processing (ICASSP 2001), vol. 4, pp. 769-773, May 2001.

[8] C. C. Martin, J. H. Winters, and N. R. Sollenberger, "Multiple-input multiple-output (MIMO) radio channel measurements," in Proc. IEEE Vehicular Technol. Conf. (Fall VTC), vol. 2, Boston, MA, Sept. 2000, pp. 774-779.

[9] J. P. Kermoal, P. E. Mogensen, S. H. Jensen, J. B. Andersen, F. Frederiksen, T. B. Sorensen, and K. I. Pedersen, "Experimental investigation of multipath richness for multi-element transmit and receive antenna arrays," in Proc. IEEE Vehicular Technol. Conf. (Spring VTC 2000), vol. 3, Tokyo, Japan, May 2000, pp. 2004-2008.

[10] G. Athanasiadou, A. Nix, and J. McGeehan, "A microcellular ray-tracing propagation model and evaluation of its narrow-band and wide-band predictions," IEEE J. Select. Areas Commun., vol. 18, pp. 322-335, Mar. 2000.

[11] G. German, Q. Spencer, A. Swindlehurst, and R. Valenzuela, "Wireless indoor channel modeling: Statistical agreement of ray tracing simulations and channel sounding measurements," in IEEE Intl. Conf. Acoustics, Speech, Signal Processing (ICASSP 2001), vol. 4, Salt Lake City, UT, May 2001, pp. 778-781.

[12] A. A. M. Saleh and R. A. Valenzuela, "A statistical model for indoor multipath propagation," IEEE J. Select. Areas Commun., vol. SAC-5, pp. 128-13, Feb. 1987

[13] Q. Spencer, B. Jeffs, M. Jensen, and A. Swindlehurst, "Modeling the statistical time and angle of arrival characteristics of an indoor multipath channel," IEEE J. Select. Areas Commun., vol. 18, pp. 347-360, Mar. 2000.

[14] A. Sayeed, "Modeling and capacity of realistic spatial MIMO channels," in IEEE Intl. Conf. Acoustics, Speech, Signal Processing (ICASSP 2001), vol. 4, Salt Lake City, UT, May 2001, pp. 765-768.

[15] T. M. Cover and J. A. Thomas, Elements of Information Theory. New York: Wiley, 1991.

[16] N. C. Giri, Multivariate Statistical Analysis. New York: Marcel Dekker, 1996.

[17] J. W. Wallace. (2002) Modeling Electromagnetic Wave Propagation in Electrically Large Structures. Brigham Young University. [Online]. Available: http://www.ycomm.org

[18] K. I. Pedersen, J. B. Andersen, J. P. Kermoal, and P. Mogensen, “A stochastic multiple-input-multiple-output radio channel model for evaluation of space-time coding algorithms," in Proc. IEEE Vehicular Technology Conf. (Fall VTC 2000), Boston, MA, Sept. 2000, pp. 893-897. 
[19] R. A. Andrews, P. P. Mitra, and R. de Carvalho, "Tripling the capacity of wireless communications using electromagnetic polarization," Nature, vol. 409, pp. 316-318, Jan. 2001.

[20] H. Bölcskei, R. U. Nabar, V. Erceg, D. Gesbert, and A. J. Paulraj, "Performance of spatial multiplexing in the presence of polarization diversity," in IEEE Intl. Conf. Acoustics, Speech, Signal Processing (ICASSP 2001), vol. 3, Salt Lake City, UT, May 2001, pp. 644-647.

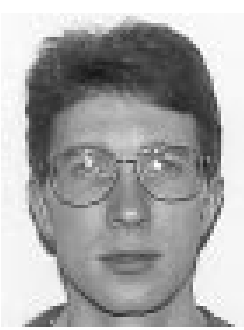

Jon W. Wallace (S'99) received the B.S. (summa cum laude) and Ph.D. degrees in electrical engineering from Brigham Young University (BYU), Provo, UT, in 1997 and 2002, respectively.

From 1995 to 1997, he worked as an Associate of Novell, Inc. in Provo. During 1997, he was a Member of the Technical Staff for Lucent Technologies, Denver, CO. From 1998 to 2002, he worked as a Graduate Student Researcher in the BYU Wireless Communications Laboratory. His research interests include wireless channel sounding and modeling, optical device modeling, and remote sensing.

Dr. Wallace received the National Science Foundation Graduate Fellowship in 1998 .

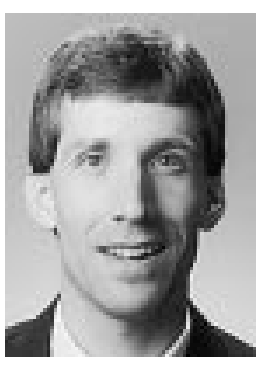

Michael A. Jensen (S'93-M'95-SM'01) received the B.S. (summa cum laude) and M.S. degrees from Brigham Young University (BYU), Provo, UT, and the $\mathrm{Ph} . \mathrm{D}$. degree, all in electrical engineering, from the University of California, Los Angeles (UCLA), in 1990, 1991, and 1994, respectively.

From 1989 to 1991, he was a graduate research assistant in the Lasers and Optics Laboratory at BYU. In 1990, he received a National Science Foundation Graduate Fellowship. From 1991 to 1994, he was a Graduate Student Researcher in the Antenna Laboratory at UCLA. Since 1994, he has been at the Electrical and Computer Engineering Department at BYU where he is currently an Associate Professor. His main research interests include antennas and propagation for personal communications, microwave circuit design, radar remote sensing, numerical electromagnetics, and optical fiber communications.

Dr. Jensen is a member of Eta Kappa Nu and Tau Beta Pi. 